\title{
Continuous Deep Sedation in the Newborn: Knowledge and Need
}

\author{
Thor Willy Ruud Hansen ${ }^{a, b}$ Dominic J.C. Wilkinson ${ }^{c, d, e}$ \\ aDepartment of Pediatrics and Adolescent Medicine, Oslo University Hospital, Oslo, Norway; ${ }^{\mathrm{b}}$ Institute of Clinical \\ Medicine, Faculty of Medicine, University of Oslo, Oslo, Norway; ' $\mathrm{Cxford}$ Uehiro Centre for Practical Ethics, Faculty \\ of Philosophy, University of Oxford, Oxford, UK; ${ }^{d}$ Murdoch Children's Research Institute, Melbourne, VIC, Australia; \\ eJohn Radcliffe Hospital, Oxford, UK
}

In the current issue of Neonatology, Dombrecht et al. [1] describe the use of continuous deep sedation (CDS) in infants dying during the first year of life in Flanders, Belgium. CDS, sometimes also referred to as "terminal sedation" or "palliative sedation," has been defined as "the monitored use of medications intended to induce a state of decreased or absent awareness (unconsciousness) in order to relieve the burden of otherwise intractable suffering" [2]. It is a well-established, albeit controversial, approach to refractory symptoms in dying patients. Dombrecht et al.'s [1] retrospective study used follow-back questionnaires and data from death certificates, covered a period of 16 months, and included the regions of Flanders and Brussels.

One key finding of the study was that CDS appeared to be occurring at a much higher rate than in previous studies in older age groups. The authors found that 39\% of neonatal and infant deaths in Belgium involved deep sedation [1]. In comparison, CDS has been reported to occur in $12-15 \%$ of adults and about $22 \%$ of paediatric deaths $[3,4]$. In a previous study in the Netherlands, it occurred prior to $19 \%$ of neonatal deaths [5].

Why is CDS occurring so often for infants in Belgium? One possibility is that CDS as reported in this study is

karger@karger.com www.karger.com/neo

Karger"
(C) 2021 The Author(s)

Published by S. Karger AG, Basel

This is an Open Access article licensed under the Creative Commons Attribution-NonCommercial-4.0 International License (CC BY-NC) (http://www.karger.com/Services/OpenAccessLicense), applicable to the online version of the article only. Usage and distribution for commercial purposes requires written permission. somewhat different from the practice in older patients. The typical setting for CDS in adults would be a patient at home or in a hospice with refractory pain or agitation despite analgesia. However, in the Dombrecht study, $82 \%$ of the infants receiving CDS died in the neonatal intensive care unit. Critically ill neonates undergoing invasive treatments such as mechanical ventilation routinely received analgesic and sedative infusions. When a decision is made to change care goals to palliation, infusions will often be continued. This does not necessarily reflect a decision to deliberately sedate to unconsciousness but perhaps more likely a desire to ensure comfort while avoiding superimposing withdrawal symptoms. In a previous study of infants dying in newborn intensive care in 4 different neonatal intensive care units, $77 \%$ received opioids, while $44 \%$ received benzodiazepines [6].

In half the cases in the Dombrecht study, the respondents reported that they did not intend to hasten death, while in 1 in 10 cases, hastening death was part of their intention. In comparison, in studies in children and adults, doctors providing CDS reported an intention to hasten death in $18-23 \%$ of cases [3, 4]. A further difference from older patients was the use of artificial nutrition and hydration. In this new study, $92 \%$ of infants receiving 
CDS had nutrition and hydration continued until death. That compares with only $54 \%$ of children and $38 \%$ of adults receiving CDS.

Dombrecht et al. [1] reflect on the lack of guidelines relating specifically to CDS in infants and newborn infants. They note that it is unclear whether guidelines for this practice in adults can be safely extrapolated to infants. We would go further. It would be highly inappropriate to apply guidance developed in relation to adult CDS to newborns. That is because newborn infants are very different from older patients, for example, in how we assess symptoms of pain and distress [7]. They are potentially ethically different from adults, for example, in the crucial role of parents in decision-making and perhaps even in the concept of a good death $[8,9]$. Finally, and importantly, it seems that CDS, at least as captured in this study, is potentially different from the practice in older patients.

It would be important to obtain a richer picture of the context and details of the care of dying infants in Belgium and reasons that sedation is used. But rather than prioritizing protocols for infant CDS, we should draw on some of the excellent existing guidelines relating to best practice palliative care and symptom management for dying infants [10]. We also need to remember the crucial importance of sensitivity to the needs and emotions, both of parents and infants. Those of us who have spent a lifetime working with sick and sometimes dying babies know that sedation is a means, but not an end, in palliative care. Whether or not an infant is sedated, love and closeness can be given and received, even in the waning hours of a short life.

\section{Acknowledgement}

The authors declare that the current study represents their joint effort and that no other individuals or entities have contributed to its content.

\section{Conflict of Interest Statement}

T.W.R.H. has no conflicts of interest to declare. He is a professor emeritus at the University of Oslo, for which he receives no honoraria or other compensation. His one-man teaching and research venture, when not working pro bono, charges honoraria on a per lecture hour basis according to the course honorarium schedule for the Norwegian Medical Association, in addition to refunds of travel expenses, none of which is related to the contents of the present commentary. D.W. has no conflicts of interest to declare. He is a member of the British Medical Association Medical Ethics Committee and the Royal College of Paediatric and Child Health Ethics and Law Advisory Committee.

\section{Funding Sources}

T.W.R.H. has no funding sources relevant to the present study. D.W. is supported for some of his research by the Wellcome Trust [203132/Z/16/Z] and by the Arts and Humanities Research Council (AHRC) as part of the UK Research and Innovation rapid response to COVID-19 [AH/V013947/1]. The funders had no role in the preparation of this manuscript or the decision to submit for publication.

\section{Author Contributions}

T.W.R.H. wrote the first draft of this manuscript, subsequently revised and amended by D.J.C.W. The authors are jointly and equally responsible for the final, submitted version.

\section{References}

1 Dombrecht L, Cools F, Cohen J, Deliens L, Goossens L, Naulaers G, et al. Continuous deep sedation until death in neonates and infants in Flanders: a post-mortem survey. Neonatology. 2021. doi: 10.1159/000517952.

2 Cherny NI, Radbruch L. Board of the European Association for Palliative Care. European Association for Palliative Care (EAPC) recommended framework for the use of sedation in palliative care. Palliat Med. 2009;23: 581-93.

3 Robijn L, Cohen J, Rietjens J, Deliens L, Chambaere K. Trends in continuous deep sedation until death between 2007 and 2013: a repeated nationwide survey. PLoS One. 2016; 11(6):e0158188.

4 Pousset G, Bilsen J, Cohen J, Mortier F, Deliens L. Continuous deep sedation at the end of life of children in Flanders, Belgium. J Pain Symptom Manage. 2011;41:449.

5 Buiting HM, Karelse MA, Brouwers HA, Onwuteaka-Philipsen $B D$, van der Heide A, van Delden JJ. Dutch experience of monitoring active ending of life for newborns. J Med Ethics. 2010;36:234-7.

6 Janvier A, Meadow W, Leuthner SR, Andrews B, Lagatta J, Bos A, et al. Whom are we comforting? an analysis of comfort medications delivered to dying neonates. J Pediatr. 2011; 159:206.

7 Hall RW, Anand KJ. Pain management in newborns. Clin Perinatol. 2014;41:895924.

8 Peck MS. Denial of the soul: spiritual and medical perspectives on euthanasia and mortality. New York, NY: Harmony Books; 1997.

9 Wilkinson D. Sleep softly: Schubert, ethics and the value of dying well. J Med Ethics. 2021;47(4):218-24.

10 Catlin A, Carter B. Creation of a neonatal end-of-life palliative care protocol. J Perinatol. 2002;22:184-95. 